\title{
Community-acquired pneumonia with infrequent presentation
}

\author{
Neumonía de la comunidad con presentación poco frecuente \\ Mario Perezpeña-Diazconti ${ }^{*}$, Sarbelio Moreno Espinosa ${ }^{2}$, Bertha Lilia Romero Baizabal ${ }^{3}$ and \\ Diana Aidee Guerrero Reséndiz ${ }^{4}$
}

${ }^{1}$ Departamento de Patología Clínica y Experimental; ${ }^{2}$ Departamento de Infectología; ${ }^{3}$ Departamento de Imagen; ${ }^{4}$ Departamento de Urgencias. Hospital Infantil de México Federico Gómez, Mexico City, Mexico

\section{Summary of the clinical history}

A case of a 3-month-old male, eutrophic height-forage, with not assessable weight because of the edema and complete vaccination scheme.

\section{Background}

A 20-year-old mother with preparatory schooling, housewife, denied tattoos, alcoholism, and smoking, apparently healthy. Father of 20 years of age, student, technical worker, denied tattoos, alcoholism, and smoking. The patient is an only son. His paternal grandfather suffers type 2 diabetes mellitus.

The patient was born and lives in Mexico City in a house of their own built with enduring materials, with all basic services. Housing with two rooms cohabited with five people. Denied coexistence with animals. He received exclusive breastfeeding. The mother declares complete immunization scheme, although immunization record was not showed.

Born of the first pregnancy, with prenatal control from the first month with nine visits, iron, and folic acid supplements. Borne after 38 weeks of pregnancy by vaginal delivery. He cried and breathed after birth. Apgar
8/9, weight $2480 \mathrm{~g}$, size $42 \mathrm{~cm}$. He was discharged with his mother 48 hours after delivery. Allergies, transfusion, traumatisms and previous hospitalizations were denied.

\section{Final condition}

The patient presented fever of $38^{\circ} \mathrm{C}$ of four days of evolution, attenuated with physical means, decreased appetite with poor suction, depositions diminished in the consistency of three days of evolution not smelling, without mucus or blood. Oligoanuria of four days of evolution was detected, of insidious, progressive onset, secondary to the decreased fluid intake. Decrease alertness of four days of evolution, with irritability, drowsiness and little response to stimuli. The patient had two previous medical consultations. Pharyngitis was diagnosed, and treatment was established with amoxicillin and acetaminophen without improvement. Leukemia was diagnosed, and the patient was then referred to the Hospital Infantil de México Federico Gómez (HIMFG).

Male patient, with a weight of $6.5 \mathrm{~kg}$, a height of $60 \mathrm{~cm}$, heart rate of $140 / \mathrm{min}$, respiratory rate $32 / \mathrm{min}$, blood pressure $95 / 50 \mathrm{mmHg}$, temperature $36.9^{\circ} \mathrm{C}$,
Correspondence:

*Mario Perezpeña-Diazconti

Email: mpdiazconti@gmail.com

2444-3409/๑ 2018. Hospital Infantil de México Federico Gómez, published by Permanyer
Available online: 25-06-2018 Bol Med Hosp Infant Mex. 2017;74:432-439 www.bmhim.com 


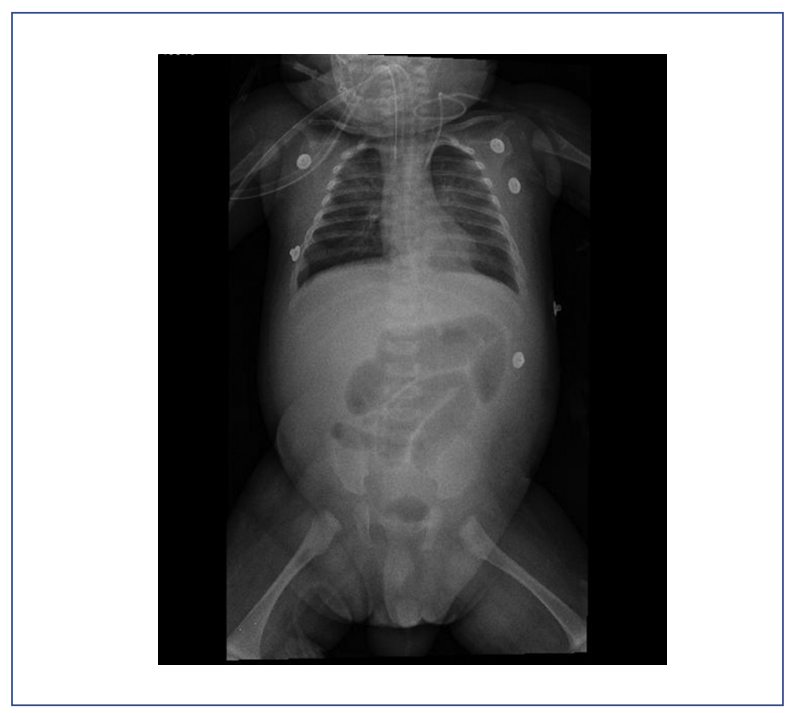

Figure 1. Anteroposterior thoracic-abdominal projection. In the parahiliar region on the left side, there are radiodensity changes that represent areas of congestion. In the abdomen, a polyhedral pattern of the intestinal loops is observed, and the liver is slightly displaced cephalically. A catheter is perceived in the right atrium. Also, a tracheal cannula and the costal arches are noticed horizontal.

capillary refill of three seconds. Physiological age similar to chronological, symmetrical, hydrated, with the paleness of skin and integuments, normocephalic skull, anterior fontanel normotensive, pupils equal, round, reactive to lights, intact tympanic membrane. Permeable nostrils, symmetric thorax with pulmonary fields with bilateral rales, predominantly basal. Rhythmic heart sounds, without murmurs. Tender and depressible abdomen, with the hepatic border of 4-3$3 \mathrm{~cm}$ and splenomegaly of $1-1-1 \mathrm{~cm}$ below the costal margin. Peristalsis diminished. Intact edematous limbs, phenotypically male genitals, testicles in both scrotal sacs. Purpuric lesions in lower and upper limbs.

Charges with physiological solution $20 \mathrm{ml} / \mathrm{kg}$ and a load of albumin were indicated, without improvement; Dobutamine, adrenaline, norepinephrine, and hydrocortisone were added. A refractory shock was diagnosed, and intubation was performed. After that, physicians started treatment with cefotaxime and vancomycin. Meningococcemia was suspected, and hemodialysis was requested. However, multiple organ failures persisted. The patient was admitted to the Pediatric Intensive Care Unit (PICU) with hematological dysfunction, cardiovascular respiratory dysfunction and disseminated intravascular coagulation (DIC). The patient's condition complicated with acute renal failure and was considered as a candidate for renal replacement. Purpura fulminans was diagnosed with tissue necrosis, small vessel thrombosis, and DIC. Based on the frequency, the presence of meningococcemia was suspected, which was treated with fresh frozen plasma, platelet concentrate, gamma globulin, vitamin $\mathrm{K}$, enoxaparin, and plasmapheresis. The patient presented persistent arterial hypotension, metabolic acidosis, purpura fulminans, DIC, multiple organ failure, hypokalemia and cardiorespiratory arrest.

\section{Imaging}

Figure 1 shows a thoracoabdominal anteroposterior projection. In the parahilar region on the left side, changes in radiodensity are observed, which represent areas of congestion, without affecting the rest of the lungs. In the abdomen, a polyhedral pattern of the intestinal loops is found, and the liver is slightly displaced cephalically. A catheter is observed in the right atrium, a tracheal cannula, and costal arches are horizontal. In the transfontanellar ultrasound, with axial, coronal and sagittal planes, showed a small ventricular system with choroidal plexuses of adequate echogenicity and situation. In the parasagittal section, the ventricular system presents decreased volume. Abdominal ultrasound shows an intestinal loop with a lost morphology, whose wall has a thickness of approximately $9 \mathrm{~mm}$ and free fluid. In the Doppler ultrasound of the pelvic limbs, permeability was detected, both in the veins and in the arteries, with no evidence of thrombosis; the trajectories of the pedis artery to the tibial artery, both arterial and venous, did not present alteration. Finally, an imaging diagnosis was made of mild cerebral edema, lungs with edema and bilateral hemorrhage, without ruling out an infectious process, free abdominal fluid, thickening of the bowel mucosa and anasarca.

\section{Discussion}

We present the case of a 3-month-old male patient, eutrophic in relation to height-for-age, with weight not assessed by edema, and with complete vaccination scheme.

The following symptomatic diagnoses can be integrated: 
- Febrile syndrome, for fever of up to $38^{\circ} \mathrm{C}$ of four days of evolution

- Diarrheal syndrome, with decreased stools consistency of three days of evolution, three times with mucus without blood

- Infectious syndrome due to fever and diarrhea described above

- Respiratory insufficiency syndrome, based on the alteration of the state of alertness that progressed to drowsiness and little response to stimuli, with gasometric criteria of respiratory acidosis, a $\mathrm{CO} 2$ pressure of $70 \mathrm{mmHg}$ and severe hypoxemia

- Capillary leak syndrome, hypoalbuminemia, and data related to fluid overload and hypotension corrected with a vasopressor agent

- Hemorrhagic syndrome of the purpuric type, based on the production of spontaneous petechiae and bruising

- Anemic syndrome, with tegument paleness, reduced hemoglobin for microcytic age according to the mean corpuscular volume

- Systemic inflammatory response syndrome, due to the presence of tachycardia, leukopenia, and fever

- Multiple organ dysfunction syndrome with cardiovascular dysfunction, by the use of vasoactive agents to maintain blood pressure and lactic acidosis twice above its normal value

- Respiratory dysfunction due to the need for invasive mechanical ventilation and hematological dysfunction with thrombocytopenia less than 11,000

Nosological diagnoses were the following:

- Septic shock, due to systemic hypoperfusion characterized by tachycardia, prolonged capillary refill, and alterations in the state of alertness with arterial tensions within percentiles, secondary to the administration of a vasopressor agent because of a clinical sepsis picture

- Heart failure with crackling rales, hepatomegaly, hemodilution, systemic hypoperfusion and inotropic therapy requirement

- Disseminated intravascular coagulation, based on purpuric lesions and disseminated intravascular coagulation ${ }^{1,2}$

- Thrombocytopenia associated with multiple organ failure, hyperlactatemia or lactic acidosis associated with global tissue hypoxia, with overproduction and a decreased clearance of lactate, secondary to mitochondrial damage

- Prerenal acute renal failure, with a glomerular filtration rate of $28 \mathrm{~mL} / \mathrm{min} / 1.73 \mathrm{~m}^{2}$, creatinine ratio of 38.5 and AKIN stage $3 .^{3}$
The patient showed clinical criteria of meningococcemia, with the presence of altered alertness, a purpuric syndrome in the skin and mucous membranes within septic shock.

Shock is characterized by a collapse in the microcirculation, with different degrees of vasoplegia and myocardial depression, according to Villeneuve et al! In the present case, cardiac output decreased gradually, which conditioned the need of inotropic support with vasoactive drugs. Even though the ejection fraction reported on echocardiography seems to be adequate, the clinical condition of the patient and the signs mentioned in the previous summary were not compatible with this ejection fraction data, since a clear state of hypodynamic shock was obvious. It should be considered that, at the time of determining the ejection fraction, the patient already had inotropic support, which could have contributed to this apparently normal values. In 1995, Wernovsky et al. proposed the use of an inotrope score, a tool that helps to know the prognosis of the patient's mortality. ${ }^{4}$ In this case, the doses of vasoactive drugs used in this patient were unknown, but it can be inferred that the value was high, which confers a high risk of death.

Based on the presence of hepatomegaly, hemodilution and increase in the percentage of extravascular pulmonary fluid, the patient is considered to have a Profile D (Cold and Wet) heart failure, according to the Stevenson diagram of four quadrants. It can be considered that the patient was under a fluid redistribution state, with depletion of circulating intravascular volume, characteristic of shock.

Endothelial dysfunction is a fundamental aspect of septic shock, as described by Ince et al..$^{5}$ The endothelium has a fundamental role both in the regulation of homeostasis and in vasomotor control, and in the immunological function; it also forms an essential vascular barrier for solute transport and osmotic balance. The state of sepsis leads to the deregulation of these functions, which favors the capillary leak and the subsequent formation of edema. This condition was present in this patient. Capillary leakage on soft tissues was evident; we could even observe the large fluid leakage showed in the radio-opacity of the radiological sequence at the abdominal level.

When the endothelial lesion is combined with fluid leakage and low cardiac output, renal function is affected, which is aggravated by presenting a fluid overload characteristic of resuscitation, increasing the days of stay in intensive care, multiple organic failure, and higher mortality. 
Conventional therapies used for the treatment of sepsis can also produce endothelial injury. For example, the use of vasoactive agents adds to endothelial dysfunction and promotes an increase in glucose levels, which in turn adversely affects the endothelium itself. Also the use of antibiotics, such as vancomycin, which produces proinflammatory cytokines and interleukin 6 (IL-6), that contributes to endothelial injury and organ failure. ${ }^{5}$

Among the complications and, at the same time, variants of multiple organ failure is that associated with thrombocytopenia, which has been described with lesions of at least three organs (in this case cardiovascular, respiratory, renal dysfunction, and possibly neurological). The decrease in the patient's platelet count caused disseminated microvascular thrombosis, which led to ischemia and increased dysfunction. ${ }^{2}$

The purpuric lesions immediately led to suspicion of meningococcemia, a condition of extreme severity. However, it is convenient to think about other etiological possibilities. $^{6}$

The characteristic lesions of purpura fulminans are erythematous lesions greater than $2 \mathrm{~mm}$ until their color turn purplish on the center, which does not disappear upon acupressure. They are indurated, painful with palpation and secondary to capillary extravasation, progressing to necrosis and with the presence of thrombosis in the microcirculation. They are presented with DIC analytical data. Of the three clinical forms in which it usually manifests itself, it is most likely in this case was in the context of a severe acute infection. This necrosis can extend to lungs, kidney, adrenal glands and even bone, which contribute to morbidity and mortality up to $50 \%$. Its diagnosis is based on the measurement of fibrinogen, D-dimer, platelets, prothrombin time (PT), partial thromboplastin time (PTT), protein $\mathrm{C}$, protein $\mathrm{S}$ and antithrombin III.

As for the agents associated with purpura fulminans are meningococcus, pneumococcus, Haemophilus influenzae, enterobacteria, Pseudomonas aeruginosa, including Pasteurella multocida, among others, which invade the wall and vascular lumen, producing septic emboli, vasculitis, thrombocytopenia, and DIC. The antibiotic considered of the first line must be at least a third-generation cephalosporin.

There are other dermatological entities that resemble purpura fulminans. Such is the case of gangrenous eczema, which is a rare infection of the skin caused mainly by Pseudomonas aeruginosa, but which can also occur due to Staphylococcus aureus or group A beta-hemolytic streptococcus. It is associated with primary and secondary immunodeficiency, but it can also occur in immunocompetent patients, whose most important risk factor is the age less than one year. Given the progressive presentation in this patient, it should be ruled out, since the lesions can be confused, although they present as erythematous, scaly, purple macules that rapidly change to papulo-vesicles or hemorrhagic and crusted blisters; if the patient survives long enough, they evolve to deep ulcers with a necrotic bottom.

About to the use of steroids in septic shock, controversy still exists today. Its use is reserved for special cases such as in the antecedent of their previous administration. In this case, faced with a suspicion of meningococcemia, the British NICE guidelines (National Institute for Health and Care Excellence)-updated in 2015-mention the possibility of using steroids at low doses: hydrocortisone $25 \mathrm{mg} / \mathrm{m}^{2}$ every six hours in the adult patient, without clear evidence of their administration in septic shock with meningococcemia in the pediatric patient.

Finally, shock and multiple organ dysfunction was extended, which caused a very severe cell dysfunction that manifested as a progressively high hyperlactatemia, which is perpetuated in an acidic environment. ${ }^{7}$ This is enough to decrease cardiac contractility, cardiac output, blood pressure and tissue perfusion, sensitizes the myocardium to the presence of arrhythmias and attenuates the cardiovascular response to catecholamines.

The final clinical diagnoses were the following:

- Refractory septic shock

- Multiple organic dysfunction

- Disseminated intravascular coagulation

- Purpura fulminans

\section{Pathology}

The case is presented based on the clinical diagnosis of meningococcemia. It is necessary to point out that we had evidence of the presence of meningococcus eleven days after the post-mortem study, verified with cultures of the autopsy rooms, the table, and some utensils. The risk for health personnel in contact with this type of patient should be considered. ${ }^{8}$ At the beginning of the study, fluid was found in the two pleural cavities, $115 \mathrm{ml}$ of serous fluid on the right side and $120 \mathrm{ml}$ on the left side, as well as $105 \mathrm{ml}$ in the pericardial cavity and $150 \mathrm{ml}$ in the abdominal cavity. The brain was found with weight gain, with edema; the 


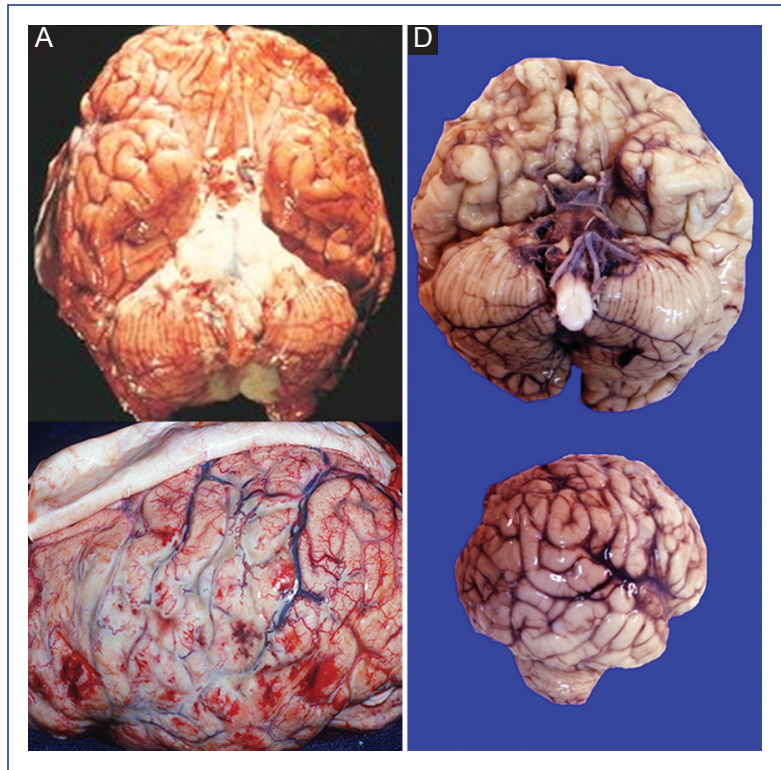

Figure 2. Comparison of the view of the base of the brain and the lateral face of a patient with meningococcal meningitis, where there is abundant purulent material, greenish grey and intense vascular congestion (A), with the patient referred in this case (B).

meninges showed a bright surface, with no evidence of meningitis (compared with the photographs of a case of meningococcal meningitis that show important edema, congestion, areas of hemorrhage and plaques of purulent material). Because of the weight of this purulent material it accumulates importantly at the base of the brain (Fig. 2A).

In our patient's brain, these changes were not observed (Fig. 2B). The histological sections were stained with hematoxylin and eosin showing a clear difference between the grey and white substance, without collections of any material; histologically, hypoxic changes were observed. In the ependymal cells, the lateral ventricles do not show any material. In a patient with meningococcal meningitis, the meninges are very thickened with abundant inflammatory infiltrate mainly composed of polymorphonuclear leukocytes, intense vascular congestion and infiltration of polymorphonuclear leukocytes into the brain parenchyma, which is not observed in our patient (Fig. 3). In the hypophysis and the choroid plexuses, there is congestion, without inflammatory infiltrate (Fig. 2).

The adrenal glands showed an adequate weight for the patient's age; patients with meningococcemia show severe hemorrhagic necrosis that affects the entire gland. The above was not observed in our patient

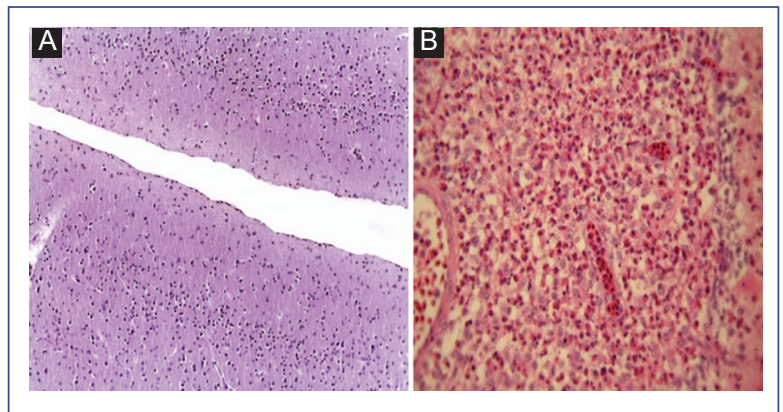

Figure 3. Histological sections of the referred patient that show an absence of inflammatory infiltrate (A). On the contrary, the intense inflammatory infiltrates of polymorphonuclear leukocytes is shown in a case of meningitis (B).

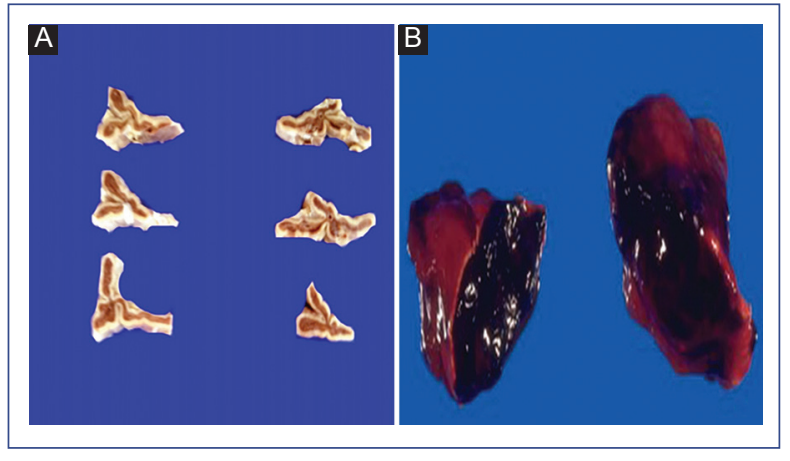

Figure 4. Histological sections of the patient's adrenal glands that do not show alterations (A). In contrast, the adrenal glands of a patient with meningococcemia are observed. Both adrenal glands are increased in weight and size and show hemorrhagic necrosis (B).

(Fig. 4A). The histological sections of the two adrenals show cortex and medulla, right and left, with a bit of congestion in the medulla. In cases of meningococcemia, we expect hemorrhage in almost all the adrenal gland (Fig. 4B). Based on these findings, we can say that the patient did not present any macroscopic data that suggests the presence of meningococcus.

The lungs were increased in weight and size; the lung cut surface has a solid appearance and areas of reddish-brown color that affect practically all the lung parenchyma. Histologically, thickening of the wall is observed. The alveolar spaces are occupied by cellular detritus, polymorphonuclear leukocytes, and erythrocytes. No bacterial colonies were observed. However, in the cultures, Pseudomonas aeruginosa developed, as in the study reported by Zhang et al. ${ }^{9}$ (Fig. 5). 
Table 1. Final anatomical diagnoses

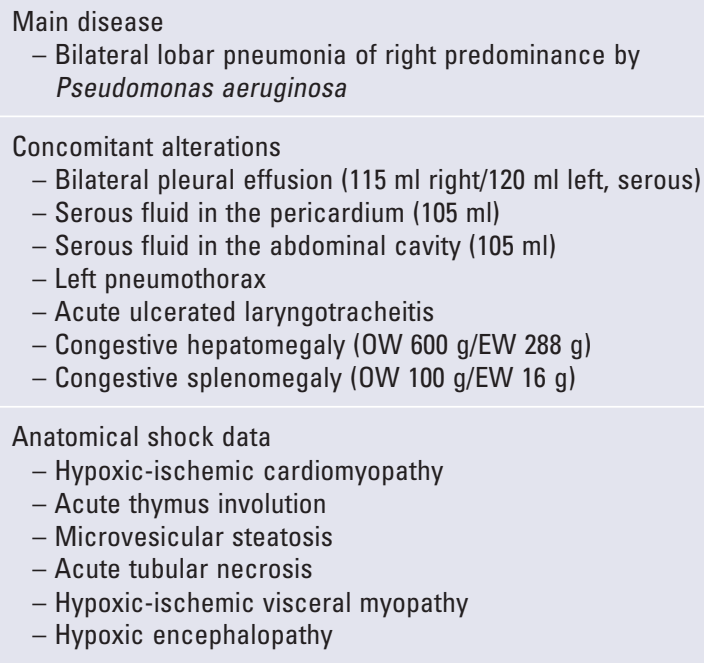

The liver was enlarged, weighed $600 \mathrm{~g}$ for an expected $188 \mathrm{~g}$, with sinusoids dilatation and congestion in addition to microvesicular steatosis. The spleen was also increased in size and weight due to congestion, with changes associated with heart failure and the final shock. Both kidneys showed adequate weight-for-age, with necrosis data due to the loss of the epithelium lining of the tubules. The heart shows shock evidence with vacuolation of the myocytes and areas of hemorrhage. We observed the esophagus and stomach in the digestive tract; the small intestine and colon were histologically intact, with their folds conserved and no alterations observed; the presence of contraction bands was observed due to shock. No DIC data were found. The bone marrow presented three series with normal maturation, practically normal cellularity and recent hemorrhage.

With all these findings, a diagnosis of bilateral lobar pneumonia with a predominance of Pseudomonas aeruginosa was made (Table 1). In all organs with autopsy culture, including the meninges, the growth of Pseudomonas aeruginosa was detected. In the blood culture, Citrobacter sedlakii was also detected.

The patient presented community pneumonia due to Pseudomonas aeruginosa ${ }^{10}$, that evolved to septic shock with the changes already described. Based on this and taking into account that the case history was pointing clearly to meningococcemia, ${ }^{11,12}$ several autopsies were reviewed (two years ago), where 126 autopsies were found in the department concerning pneumonia data. In 47 of these autopsies $(37 \%)$ the diagnosis of pneumonia was made; 22 female patients and 25 male. The age range was very broad, from five

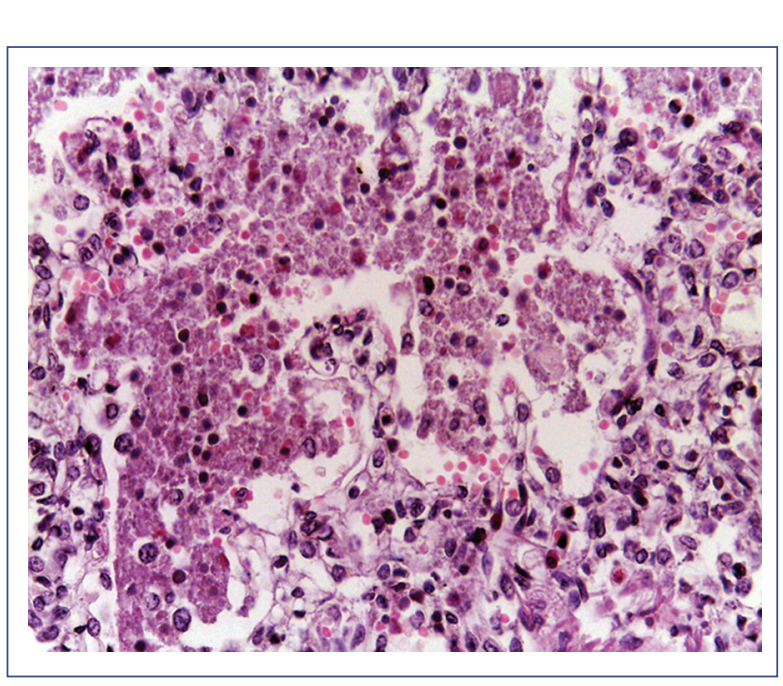

Figure 5. Histological sections of the lungs that show inflammatory infiltrate of polymorphonuclear leukocytes and macrophages, some with hemophagocytosis.

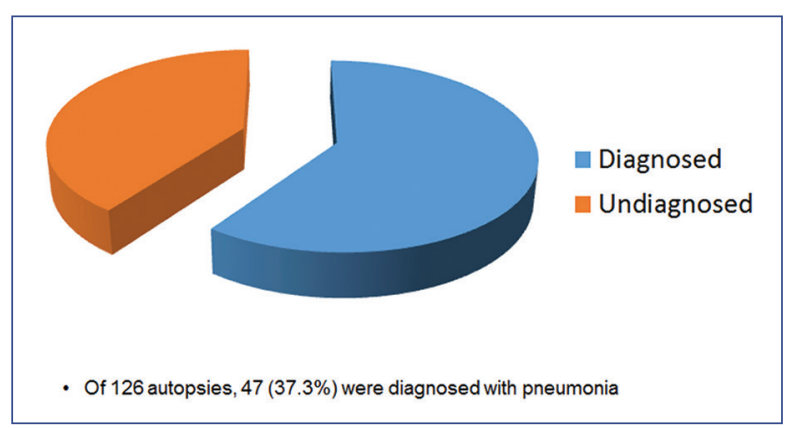

Figure 6. Data on autopsies and pneumonia.

days to 15 years of age (Fig. 6). Cases of pneumonia with clinical diagnosis at the time of autopsy accounted for $60 \%$. In $40 \%$ of these cases, the diagnosis of pneumonia was not suspected when the patient was alive. Patients with primary immunodeficiencies such as granulocytosis, idiopathic intractable diarrhea, severe combined immunodeficiency, neoplasms such as osteosarcoma, neuroblastoma, leukemia, and other infections such as cytomegalovirus, salmonellosis, Epstein-Barr virus, and varicella were found. The causes of death included mainly shock, septic shock, mixed shock and hypovolemic shock; as a second cause, sepsis. In four cases, the main disease and the cause of death was pneumonia. Finally, most of these autopsies $(68 \%)$ could not isolate any microorganism. In the rest of the autopsies, the isolation of bacteria such as Enterobacter and Escherichia coli 


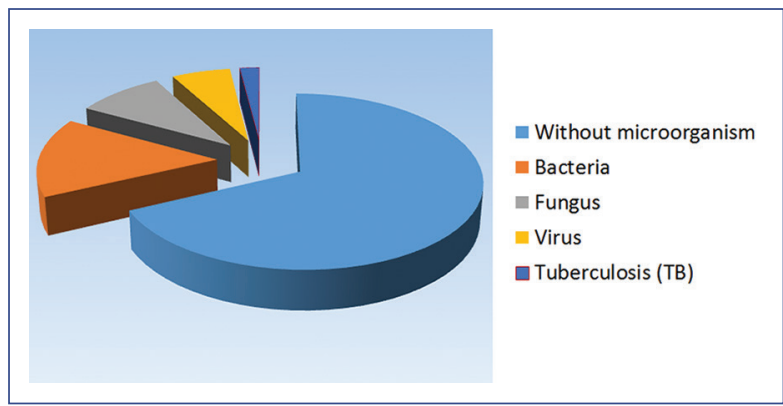

Figure 7. Of 126 autopsies, no microorganism was isolated in $68 \%$; bacteria were isolated in $15 \%$; fungi in $6 \%$, virus in $6 \%$ and tuberculosis in $2 \%$.

(15\%) was achieved. Fungi, mainly Aspergillus and Candida, were isolated in $9 \%$. Viruses, such as respiratory syncytial virus and cytomegalovirus, were detected in $6 \%$, and in $2 \%$, miliary tuberculosis was detected, which in life was diagnosed as Mycoplasma (Fig. 7).

The objective of these data is to promote the importance of autopsies. In the case presented, everything seemed to indicate that the diagnosis was meningococcemia. However, after the autopsy, sepsis was confirmed by Pseudomonas aeruginosa. The finding of $40 \%$ of cases of pneumonia that were not diagnosed while the patient was alive is very important, as well as finding atypical cases of pneumonia (for example, Nocardia). There are very interesting examples of pneumonia by different microorganisms that could not be detected without an autopsy (Fig. 7).

\section{Final comments}

\section{Pediatric Intensive Care Unit}

At the moment in which the patient arrives, the manifested symptoms are very important. The patient in the reported case had purpura fulminans syndrome associated with thrombocytopenia and organic dysfunctions. The present case is an example of how complicated cases are not recognized in the health system. Treatment in intensive care includes heparin (enoxaparin may be used, although the results are controversial since it may increase the risk of bleeding in young children). Patients with purpura fulminans syndrome often have deficiencies of the Von Willebrand factor polymer proteinase, ${ }^{13}$ which can be treated with early plasmapheresis until coagulation is normalized, besides it would help to control the inflammatory response. The next treatment is early hemofiltration, with which mortality is reduced to $25-30 \%$ since both techniques remove the excess of coagulation factors, fibrinogen inhibitors, and inflammatory mediators. As a result, the patient stabilizes.

\section{Clinical laboratory}

In this case, all cultures made to the patient were positive for Pseudomonas aeruginosa. In the blood cultures, there was isolation after 12 hours. Following the standards, the susceptibility pattern characteristic of the pathogen is the M100DCLI guide.

\section{Laboratory of intestinal bacteriology}

Pseudomonas aeruginosa are Gram-negative non-fermenting bacilli, large colonies with irregular edges, and present beta-hemolysis and pigments. ${ }^{14}$ In particular, the strains isolated in this case were small and irregular and presented beta-hemolysis at more than 24 hours up to almost 48 hours. Another peculiarity of this bacterium is that it produces biofilms, a characteristic shared by many microorganisms. Worldwide, Pseudomonas aeruginosa has the presence of an epidemic strain, ${ }^{15}$ multiresistant, panresistant and sensitive. Under stressful conditions, the bacteria induce high production of virulence factors. ${ }^{16}$ In a study conducted in England, it was shown that a particular stain is spreading and has caused deaths, being sensitive and community-acquired. ${ }^{17}$

\section{Ethical disclosures}

Protection of human and animal subjects. The authors declare that no experiments were performed on humans or animals for this study.

Confidentiality of data. The authors declare that they have followed the protocols of their work center on the publication of patient data.

Right to privacy and informed consent. The authors declare that no patient data appear in this article.

\section{Conflict of interest}

Authors declare no conflicts of interest.

\section{References}

1. Villeneuve A, Joyal JS, Proulx F, Ducruet T, Poitras N, Lacroix J. Multiple organ dysfunction syndrome in critically ill children: clinical value of two lists of diagnostic criteria. Ann Intensive Care. 2016;6:40. 
2. Di Nisio M, Thachil J, Squizzato A. Management of disseminated intravascular coagulation: A survey of the International Society on Thrombosis and Haemostasis. Thromb Res. 2015;136:239-42.

3. Bagshaw SM, George C, Bellomo R, ANZICS Database Management Committee. A comparison of the RIFLE and AKIN criteria for acute kidney injury in critically ill patients. Nephrol Dial Transplant. 2008;23: 1569-74

4. Gaies MG, Gurney JG, Yen AH, Napoli ML, Gajarski RJ, Ohye RG, et al Vasoactive-inotropic score as a predictor of morbidity and mortality in infants after cardiopulmonary bypass. Pediatr Crit Care Med. 2010;11: 234-8.

5. Ince C, Mayeux PR, Nguyen T, Gomez H, Kellum JA, Ospina-Tascón GA et al. The endothelium in sepsis. Shock. 2016;45:259-70.

6. Aroor S, Chaitanya V, Suneel CM. Purpura fulminans in a child: a case report. J Clin Diagnostic Res. 2012;6:1812-3.

7. Ingelfinger JR, Kraut JA, Madias NE. Lactic acidosis. N Engl J Med 2014;371:2309-19.

8. Nagel FW, Ezeoke I, Antwi M, Del Rosso PE, Dorsinville M, Isaac BM, et al. Delayed recognition of fatal invasive meningococcal disease in adults. JMM Case Rep. 2016;3:e005027.

9. Zhang Q, Smith JC, Zhu Q, Guo Z, MacDonald NE. A five-year review of Pseudomonas aeruginosa bacteremia in children hospitalized at a single center in southern China. Int J Infect Dis. 2012;16:e628-32.
10. Huang YC, Lin TY, Wang $\mathrm{CH}$. Community-acquired Pseudomonas aeruginosa sepsis in previously healthy infants and children: analysis of forty-three episodes. Pediatr Infect Dis J. 2002;21:1049-52.

11. Lécuyer $H$, Borgel $D$, Nassif $X$, Coureuil M. Pathogenesis of meningococcal purpura fulminans. Pathog Dis. 2017;75.

12. Vaz LE. Meningococcal disease. Pediatr Rev. 2017;38:158-69.

13. Kuo KC, Kuo HC, Huang LT, Lin CS, Yang SN. The clinical implications of $\mathrm{ABO}$ blood groups in Pseudomonas aeruginosa sepsis in children. J Microbiol Immunol Infect. 2013;46:109-14.

14. Murray P, Rosenthal K, Pfaller M. Microbiología médica. Madrid: Elsevier 2015.

15. López Causapé C, Rojo ME, Mulet X, Cabot G, Moya B, Figuerola J, et al. Clonal dissemination, emergence of mutator lineages and antibiotic resistance evolution in Pseudomonas aeruginosa cystic fibrosis chronic lung infection. PLoS One. 2013;8:e71001.

16. Aguilar-Rodea $P, Z$ Zúñiga G, Rodríguez-Espino BA, Olivares Cervantes AL, Gamiño Arooyo AE, Moreno-Espinosa S, et al. Identification of extensive drug resistant Pseudomonas aeruginosa strains: New clone ST1725 and high-risk clone ST233. PLoS One. 2017;12:e0172882.

17. Mudau M, Jacobson R, Minenza N, Kuonza L, Morris V, Engelbrecht H, et al. Outbreak of multi-drug resistant Pseudomonas aeruginosa bloodstream infection in the haematology unit of a South African Academic Hospital. PLoS One 8. 2013;8:e55985. 